\title{
HIGHER ORDER COMPOSITION RUNGE-KUTTA METHODS
}

\author{
D. J. L. CHEN, J. C. CHANG, C. H. CHENG
}

\begin{abstract}
We derive a fifth order five-stage explicit Runge-Kutta composition method, and an error estimator using linear combination of stage values and output values over two steps. Numerical results presented by testing the new pair over DETEST problems show a significant improvement.
\end{abstract}

\section{Introduction}

All explicit Runge-Kutta methods of order higher than four need more integration stages than their order [3]. The minimum stages for various orders are shown in Table 1.

Table 1. Minimum number of stages for various orders

\begin{tabular}{|l|l|l|l|l|l|l|l|l|l|l|}
\hline Order & 1 & 2 & 3 & 4 & 5 & 6 & 7 & 8 & 9 & 10 \\
\hline Minimum Stages & 1 & 2 & 3 & 4 & 6 & 7 & 9 & 11 & 12 & 13 \\
\hline
\end{tabular}

There have been several attempts to break this barrier(Butcher barrier). The first one is the explicit type of effective order methods by Gifkins [14]. By perturbing the initial value $y_{0}$ into $y_{0}+\alpha_{1} h y_{0}^{\prime}+\alpha_{2} h^{2} y_{0}^{\prime \prime}+\cdots+\alpha_{p} h^{p} y_{0}^{(p)}$, the effective order methods offer several free parameters to attain higher order or to have better stability behavior. The second one is so-called Almost Runge-Kutta methods (ARK) [6]. There are three quantities, $y_{0}, h y_{0}^{\prime}, h^{2} y_{0}^{\prime \prime}$, passing from step to step. Even though the third quantity $h^{2} y_{0}^{\prime \prime}$ is of lower order, the contributed errors cancel out using annihilation conditions. Having more information passing from step to step, ARKs have cheaper error estimators, dense output and higher attainable order for explicit form.

Instead of using higher derivative terms to break the Butcher barrier, we propose to use the idea of composition methods. If the second method cancel out the principal local truncation error of the first method, then the composition of the two methods presents one order higher feature. The main purpose of this paper is to exploit the order conditions of these composition methods and to implement the underlying methods.

Received November 17, 2006.

2000 Mathematics Subject Classification. Primary 40B05.

Key words and phrases. The $C$ and $D$ simplifying assumption, elementary weight function, composition rule, linear combination of trees. 
In section 2, the basic theory of algebraic approach for integration methods, by Butcher 1972 [5], is presented. In section 3, the conditions imposed on the coefficients to achieve the cancellation of principal local truncation errors between two methods are derived. We derive a fifth order composition method of two fourth order explicit RK with stage number five. In section 4, some preliminary comparisons are presented between new methods and several existing methods (famous Runge-Kutta method RK54 and Dormand and Prince(5,4)).

It is our hope that not only this new pair five-stage methods presents order five feature, but also competes with the existing methods.

\section{Recursive derivation of order conditions}

In the paper, the initial value problems are considered as in autonomous form,

$$
y^{\prime}(x)=f(y(x)), \quad y\left(x_{0}\right)=y_{0},
$$

where $f: \mathbb{R}^{n} \rightarrow \mathbb{R}^{n}$ is differentiable to an arbitrary order. In 1972 Butcher [5] proposed a way of deriving the coefficients that occur in Taylor series expansions of the exact and numerical solutions. In Butcher's approach that Taylor series expansion of the exact solution $y(x+h)$ about $x$ can be expressed as

$$
y(x+h)=y(x)+\sum_{t \in T} \frac{1}{\sigma(t) \gamma(t)} F(t)(y(x)) h^{r(t)},
$$

where $r(t)$ is the order of the tree $t$, the symmetry $\sigma(t)$ and density $\gamma(t)$ are real valued functions defined on the rooted trees $T$ [7], $F(t)(y(x))$ is the elementary differential corresponding to $t$. This one-to-one interpretation of the elementary differentials and their coefficients in terms of functions defined on trees has led to an elegant algebraic approach for integration methods.

The Taylor series expansion of the numerical solution $y_{n+1}$ about $x_{n}$ is

$$
y_{n+1}=y_{n}+\sum_{t \in T} \frac{\alpha(t)}{\sigma(t)} F(t)\left(y_{n}\right) h^{r(t)},
$$

where the $\alpha(t)$ is called the elementary weight function. The function $E: T \rightarrow R$, is defined by $E(t)=\frac{1}{\gamma(t)}$, identified as the coefficient of $\frac{1}{\sigma(t)} F(t)(y(x)) h^{r(t)}$. E can be regarded as the elementary weight function of the exact solution. By comparing the Taylor series expansions of the exact solution (1) with numerical solution (2), the order conditions of a $p^{\text {th }}$ order method $\alpha$ are $\alpha(t)=E(t)$ for all $t \in T$ with $r(t) \leq p$.

In this paper, our aim is to enhance the accuracy over two methods. In order to achieve this goal, we need to consider the composite mapping $\alpha \beta$ over two steps. This can be regarded as $\alpha \beta$ which is a composition mapping of two mappings $\alpha$ and $\beta$. Butcher [7] gave the value

$$
(\alpha \beta)(t)=\beta(\phi) \alpha(t)+\beta(t)+\sum_{u<T} \alpha(t \backslash u) \beta(u), \quad \forall t \in T .
$$


where $u$ is a subtree of $t$ sharing the same root with the tree $t$ and $t \backslash u$ is the remaining part of $t$ after deleting the subtree $u$. The composition rule is widely applicable to other types of methods.

We consider the integration over stepsize $\theta h$ for exact solution. Let $E^{(\theta)}(t)$ denote the elementary weight function of exact solution over $\theta$ steps. The Taylor series of $y(x+\theta h)$ at $x$ is

$$
\begin{aligned}
y(x) & +\sum_{t \in T} \frac{E^{(\theta)}(t)}{\sigma(t)} F(t)(y(x)) h^{r(t)} \\
& =y(x)+\sum_{t \in T} \frac{E(t)}{\sigma(t)} F(t)(y(x))(\theta h)^{r(t)} \\
& =y(x)+\sum_{t \in T} \frac{E(t) \theta^{r(t)}}{\sigma(t)} F(t)(y(x)) h^{r(t)} .
\end{aligned}
$$

Compare (4) with (5), we have

$$
E^{(\theta)}(t)=E(t) \theta^{r(t)} \forall \operatorname{tin} T .
$$

In this paper, we will apply equation (6) in the case of $\theta=2$.

The algebraic conditions on the coefficients of the method become increasingly complicated when order increases. The algebraic approach based on Runge-Kutta methods to solve these conditions was proposed in [5] in 1972. In addition, there are existing some theories developed by Hairer and Wanner [16], Albrecht [1] and F. Bornemann [2] which provide other approaches to the order conditions. Here, we will make reference to Butcher theory. In the present approach, the order conditions can be written down easily by transcribing the structure of the corresponding rooted trees. Moreover, we study the conditions that the method has to satisfy to attain a prescribed order of accuracy.

As the order increases, particularly for explicit Runge-Kutta methods, the number of conditions rises rapidly and soon becomes unmanageable. It is reasonable to reduce the order conditions by simplifying assumptions.

Butcher [5] proposes the $C$ and $D$ simplifying conditions to reduce the order conditions of RK methods. The $C(n)$ conditions for Runge Kutta methods are

$$
A c^{i-1}=\frac{1}{i} c^{i}, \quad \forall i=1, \ldots n .
$$

The $D(k)$ conditions for Runge Kutta methods are

$$
b^{T} c^{i-1} A=b^{T} \frac{1}{i}\left(1-C^{i}\right), \quad \forall i=1, \ldots k .
$$

where $C=\operatorname{diag}\left(c_{1}, c_{2}, \ldots, c_{s}\right)$. For the explicit matrix $A$, the first and second components of $A c$ equal zero. Therefore, it is not possible for an explicit RK method to have $C(2)$ conditions. In order that our explicit methods mimic the $C$ (2) conditions up to order five, we adopt the simplifying techniques in Butcher (Chapter 3) [7] and linear combinations of trees in Chan [9].

The following conditions make sure that the explicit Runge-Kutta methods have the equivalent order conditions as the $C$ (2) conditions up to order five. 
(a) $\sum_{i=1}^{s} b_{i} a_{i j}=b_{j}\left(1-c_{j}\right), \quad \forall j=1, \ldots s$. (The $D(1)$ conditions )

(b) $\sum_{j=1}^{s} a_{i j} c_{j}^{k-1}=\frac{1}{k} c_{i}^{k}, \quad \forall i \neq 2, k=1,2$. (The $C(2)$ conditions)

(c) $b_{2}=0$.

(d) $\sum_{i=1}^{s} b_{i}\left(1-c_{i}\right) a_{i 2}=0$.

Using conditions (a)-(d), the 17 order conditions of a five order method can be reduced to 6 order conditions corresponding to the following trees,

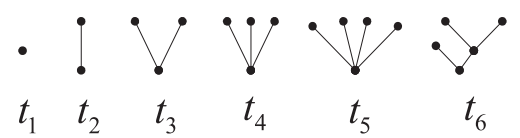

\section{An enhanced order composition methods}

In this section, a new type of enhanced order composition methods is derived through analysing the PLTE (Principal Local Truncation Error) over two steps. We also derive one order higher composition methods over two steps. The scheme of error estimation proposed in our composition method is error estimation over two steps. Compared with the traditional Runge-Kutta methods, we have more free parameters, smaller PLTE and more accurate. The conditions to derive one-order higher composition methods are investigated. A fifth order composition method (ECRK5) is proposed using two different fourth-order explicit RungeKutta methods(ECRK5_1 and ECRK5_2) with five stages.

For $p^{\text {th }}$ order methods with corresponding weight function $\alpha$ over stepsize $\theta h$, the general order conditions in Butcher [7] is

$$
\alpha^{(\theta)}(t)=E^{(\theta)}(t)=\frac{\theta^{r(t)}}{\gamma(t)}, \quad \forall t: r(t) \leq p .
$$

With the aid of this condition, we have the following theorem.

Theorem 1. Let $\alpha$ and $\beta$ be of order $p$ over stepsize $h$, then $\alpha \beta$ is of order $p+1$ over stepsize $2 h$ if and only if

$$
\alpha(t)+\beta(t)=\frac{2}{\gamma(t)}, \quad \forall t, r(t)=p+1
$$

Proof. $\alpha$ and $\beta$ satisfy the order conditions

$$
\alpha(t)=\beta(t)=E(t), \quad \text { for all } r(t) \leq p .
$$

The composition rules of $\alpha \beta$ and $E^{(2)}$ are

$$
\alpha \beta(t)=\alpha(t)+\beta(t)+\sum_{u<t} \alpha(t \backslash u) \beta(u)
$$




$$
E^{(2)}(t)=E E(t)=E(t)+E(t)+\sum_{u<t} E(t \backslash u) E(u)
$$

“ $\Longrightarrow " \alpha \beta$ is of order $p+1, \alpha \beta(t)=E^{(2)}(t) \forall r(t) \leq p+1$. Because each member of $t \backslash u$ and $u$ are of order less than or equal to $p$, and $\alpha, \beta$ are of order $p$, we know that

$$
\alpha(t \backslash u)=E(t \backslash u), \beta(u)=E(u) .
$$

By comparing (8) and (9), we have $\alpha(t)+\beta(t)=E(t)+E(t)=\frac{2}{\gamma(t)}$.

“ $\Longleftarrow$ " For trees of order $p+1$, the composition rule of $\alpha \beta$

$$
\begin{aligned}
\alpha \beta(t) & =\alpha(t)+\beta(t)+\sum_{u<t} \alpha(t \backslash u) \beta(u) \text { (From (8)) } \\
& =\frac{2}{\gamma(t)}+\sum_{u<t} E(t \backslash u) E(u)(\text { From (10)) } \\
& =E(t)+E(t)+\sum_{u<t} E(t \backslash u) E(u)=E^{(2)}(t) \text { (From (9)) }
\end{aligned}
$$

Therefore, $\alpha \beta(t)=E^{(2)}(t) \forall r(t) \leq p+1$, together with equation (7), we have $\alpha \beta$ is of order $p+1$.

Example 1. In this example, we will derive a fifth order composition method of $(A, b, c)_{5}$ with the corresponding elementary weight function $\alpha(t)$,

\begin{tabular}{c|ccccc}
0 & 0 & 0 & 0 & 0 & 0 \\
$c_{2}$ & $a_{21}$ & 0 & 0 & 0 & 0 \\
$c_{3}$ & $a_{31}$ & $a_{32}$ & 0 & 0 & 0 \\
$c_{4}$ & $a_{41}$ & $a_{42}$ & $a_{43}$ & 0 & 0 \\
1 & $a_{51}$ & $a_{52}$ & $a_{53}$ & $a_{54}$ & 0 \\
\hline & $b_{1}$ & 0 & $b_{3}$ & $b_{4}$ & $b_{5}$
\end{tabular}

$(\bar{A}, \bar{b}, \bar{c})_{5}$ with the corresponding elementary weight function $\beta(t)$,

\begin{tabular}{c|ccccc}
0 & 0 & 0 & 0 & 0 & 0 \\
$\bar{c}_{2}$ & $\bar{a}_{21}$ & 0 & 0 & 0 & 0 \\
$\bar{c}_{3}$ & $\bar{a}_{31}$ & $\bar{a}_{32}$ & 0 & 0 & 0 \\
$\bar{c}_{4}$ & $\bar{a}_{41}$ & $\bar{a}_{42}$ & $\bar{a}_{43}$ & 0 & 0 \\
1 & $\bar{a}_{51}$ & $\bar{a}_{52}$ & $\bar{a}_{53}$ & $\bar{a}_{54}$ & 0 \\
\hline & $\bar{b}_{1}$ & 0 & $\bar{b}_{3}$ & $\bar{b}_{4}$ & $\bar{b}_{5}$
\end{tabular}

In order that our methods have $D(1)$ condition and mimic the $C(2)$ conditions up to order five, the new methods satisfy conditions (a) to (d) on page 202.

The procedures to determine the coefficients of these methods are shown as follows: 
Step 1: Solving for the order conditions on $t_{2}$ to $t_{5}$ for these two methods, we have

$$
\left(\begin{array}{l}
b_{3} \\
b_{4} \\
b_{5}
\end{array}\right)=\left(\begin{array}{lll}
c_{3} & c_{4} & 1 \\
c_{3}^{2} & c_{4}^{2} & 1 \\
c_{3}^{3} & c_{4}^{3} & 1
\end{array}\right)^{-1}\left(\begin{array}{c}
\frac{1}{2} \\
\frac{1}{3} \\
\frac{1}{4}
\end{array}\right),\left(\begin{array}{c}
\bar{b}_{3} \\
\bar{b}_{4} \\
\bar{b}_{5}
\end{array}\right)=\left(\begin{array}{ccc}
\bar{c}_{3} & \bar{c}_{4} & 1 \\
\bar{c}_{3}^{2} & \bar{c}_{4}^{2} & 1 \\
\bar{c}_{3}^{3} & \bar{c}_{4}^{3} & 1
\end{array}\right)^{-1}\left(\begin{array}{c}
\frac{1}{2} \\
\frac{1}{3} \\
\frac{1}{4}
\end{array}\right) .
$$

and for the order conditions on $t_{1}$, we have $b_{1}=1-b_{3}-b_{4}-b_{5}$ and $\bar{b}_{1}=1-\bar{b}_{3}-$ $\bar{b}_{4}-\bar{b}_{5}$.

Step 2: For the $C$ (2) conditions except for the second components on page 202 (b), we have

$$
\begin{aligned}
& a_{32}=\frac{c_{3}^{2}}{2 c_{2}}, \quad \bar{a}_{32}=\frac{\bar{c}_{3}^{2}}{2 \bar{c}_{2}}, \\
& a_{31}=c_{3}-a_{32}, \quad \bar{a}_{31}=\bar{c}_{3}-\bar{a}_{32}, \\
& a_{42}=\frac{b_{3}\left(1-c_{3}\right) a_{32}}{b_{4}\left(1-c_{4}\right)}, \quad \bar{a}_{42}=\frac{\bar{b}_{3}\left(1-\bar{c}_{3}\right) \bar{a}_{32}}{\bar{b}_{4}\left(1-\bar{c}_{4}\right)}, \\
& a_{43}=\frac{\frac{1}{2} c_{4}^{2}-a_{42} c_{2}}{c_{3}}, \quad \bar{a}_{43}=\frac{\frac{1}{2} \bar{c}_{4}^{2}-\bar{a}_{42} \bar{c}_{2}}{\bar{c}_{3}}, \\
& a_{41}=c_{4}-a_{42}-a_{43}, \quad \bar{a}_{41}=\bar{c}_{4}-\bar{a}_{42}-\bar{a}_{43} .
\end{aligned}
$$

Step 3: For the $D(1)$ conditions on page 202 (a), we have

$$
\begin{aligned}
\left(a_{51}, a_{52}, a_{53}, a_{54}, 0\right) & =\frac{b^{T}(I-C)-b_{3} A[3]-b_{4} A[4]}{b_{5}}, \\
\left(\bar{a}_{51}, \bar{a}_{52}, \bar{a}_{53}, \bar{a}_{54}, 0\right) & =\frac{\bar{b}^{T}(I-\bar{C})-\bar{b}_{3} \bar{A}[3]-\bar{b}_{4} \bar{A}[4]}{\bar{b}_{5}},
\end{aligned}
$$

where $A[i]$ and $\bar{A}[i]$ are the $i^{\text {th }}$ row of matrix $A$ and $\bar{A}$ respectively, and $I$ is the identity matrix, $C=\operatorname{diag}(c)$ and $\bar{C}=\operatorname{diag}(\bar{c})$.

Our methods are of order four by solving the above conditions.

Step 4: In order that our new composite method is of order five, we need consider $\alpha(t)+$ $\beta(t)=\frac{2}{\gamma(t)}$ on trees $t_{5}$ and $t_{6}$.

(1) For the bushy tree $t_{5}$, the order condition can be simplified to

$$
\begin{aligned}
& b^{T} c(c-1)\left(c-c_{3}\right)\left(c-c_{4}\right)=-\frac{1}{20}+\frac{c_{3}+c_{4}}{12}-\frac{c_{3} c_{4}}{6}, \\
& \bar{b}^{T} \bar{c}(\bar{c}-1)\left(\bar{c}-\bar{c}_{3}\right)\left(\bar{c}-\bar{c}_{4}\right)=-\frac{1}{20}+\frac{c_{3}+c_{4}}{12}-\frac{c_{3} c_{4}}{6},
\end{aligned}
$$

based on

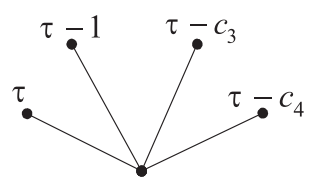


Since the equations $b^{T} c(c-1)\left(c-c_{3}\right)\left(c-c_{4}\right)=\bar{b}^{T} \bar{c}(\bar{c}-1)\left(\bar{c}-\bar{c}_{3}\right)\left(\bar{c}-\bar{c}_{4}\right)=0$, we have

$$
\begin{aligned}
& b^{T} c(c-1)\left(c-c_{3}\right)\left(c-c_{4}\right)+\bar{b}^{T} \bar{c}(\bar{c}-1)\left(\bar{c}-\bar{c}_{3}\right)\left(\bar{c}-\bar{c}_{4}\right) \\
= & \left(-\frac{1}{20}+\frac{c_{3}+c_{4}}{12}-\frac{c_{3} c_{4}}{6}\right)+\left(-\frac{1}{20}+\frac{\bar{c}_{3}+\bar{c}_{4}}{12}-\frac{\bar{c}_{3} \bar{c}_{4}}{6}\right)=0 .
\end{aligned}
$$

(2) The order condition on the linear combination of tree $t_{6}$

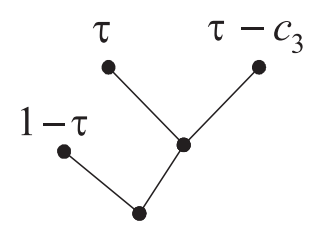

is used to simplify the calculation work. Because the equation $b^{T}(1-c) A c(c-$ $\left.c_{3}\right)=\bar{b}^{T}(1-\bar{c}) \bar{A} \bar{c}\left(\bar{c}-\bar{c}_{3}\right)=0$, and this is equivalent to the conditions:

$$
\begin{aligned}
& b^{T}(1-c) A c\left(c-c_{3}\right)=\frac{1}{60}-\frac{c_{3}}{24}, \\
& \bar{b}^{T}(1-\bar{c}) \bar{A} \bar{c}\left(\bar{c}-\bar{c}_{3}\right)=\frac{1}{60}-\frac{\bar{c}_{3}}{24} .
\end{aligned}
$$

Therefore, we have

$$
b^{T}(1-c) A c\left(c-c_{3}\right)+\bar{b}^{T}(1-\bar{c}) \bar{A} \bar{c}\left(\bar{c}-\bar{c}_{3}\right)=\frac{1}{60}-\frac{c_{3}}{24}+\frac{1}{60}-\frac{\bar{c}_{3}}{24}=0
$$

then

$$
c_{3}+\bar{c}_{3}=\frac{4}{5}
$$

(3) By using (11) and (12), we obtain

$$
\bar{c}_{3}=\frac{4}{5}-c_{3}, \text { and } \bar{c}_{4}=\frac{2-5 c_{4}+10 c_{3} c_{4}}{-3+10 c_{3}} .
$$

There are four free parameters $c_{2}, c_{3}, c_{4}, \bar{c}_{2}$ left in our approach. These free parameters are chosen in order that the PLTE of the composition method is reduced, and all the abscissae are between 0 and 1 . We consider some trees of order six :

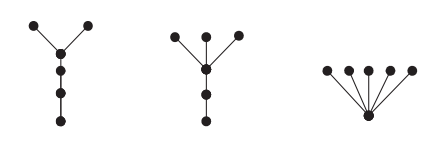

Using simplifying technique to solve $t_{7}, t_{8}$, $t_{9}$, we obtain the value of these free parameters. 
(1) For tree $t_{7}$, the $\alpha \beta\left(t_{7}\right)-\frac{2^{6}}{360}$ can be simplified to

$$
\begin{aligned}
y_{1}= & b^{T} A^{3} c\left(c-c_{2}\right)+\bar{b}^{T} \bar{A}^{3} \bar{c}\left(\bar{c}-\bar{c}_{2}\right)+b^{T} A^{2} c\left(c-c_{2}\right) \\
& +\frac{1}{24}+\frac{1}{18}+\frac{1}{24}+2 \bar{b}^{T} \bar{A}^{3} \bar{c}-\frac{2^{6}}{3 \times 4 \times 5 \times 6} .
\end{aligned}
$$

Solving $y_{1}=0$ for $c_{2}$, we obtain

$$
c_{2}=-\frac{-4+12 \bar{c}_{2}-15 \bar{c}_{2} c_{3}}{15 c_{3}} .
$$

(2) For tree $t_{8}$, the condition $\alpha \beta\left(t_{8}\right)-\frac{2^{6}}{120}$ is simplified to

$$
\begin{aligned}
y_{2}= & b^{T} A^{2} c^{3}+\bar{b}^{T} \bar{A}^{2} \bar{c}^{3}+b^{T} A c^{3}+3 \bar{b}^{T} \bar{A}^{2} \bar{c}^{2} \\
& +\frac{1}{8}+\frac{1}{6}+\frac{3}{24}-\frac{2^{6}}{4 \times 5 \times 6} .
\end{aligned}
$$

Solving $y_{2}=0$ for $c_{4}$, we have

$$
c_{4}=-\frac{6-65 c_{3}+50 c_{3}^{2}}{50\left(1-2 c_{3}\right)} .
$$

(3) For bushy tree $t_{9}$, the condition $\alpha \beta\left(t_{9}\right)-\frac{2^{6}}{6}$ is simplified to

$$
y_{3}=b^{T} c^{5}+\bar{b}^{T} \bar{c}^{5}+5 \bar{b}^{T} \bar{c}^{4}+1+\frac{5}{2}+\frac{10}{3}+\frac{10}{4}-\frac{2^{6}}{6} .
$$

Solving $y_{3}=0$ for $c_{3}$, we obtain

$$
c_{3}=\frac{384101}{1000000} .
$$

(4) Finally, for free parameter $\bar{c}_{2}$, we choose $\bar{c}_{2}=\frac{1}{5}$.

These methods are named ECRK5_1, and ECRK5_2 which is given in the Appendix. The error estimation for the composition method is also be derived by using a "one-step zero approximation" based on embedded technique [8]. Let $e=(1, \ldots, 1)^{T} \in \mathbb{R}^{11}$ and

$$
\delta^{T}=\left(\hat{\delta}_{1}, 0, \hat{\delta}_{3}, \hat{\delta}_{4}, \hat{\delta}_{5}, \hat{\delta}_{6}, 0, \hat{\delta}_{8}, \hat{\delta}_{9}, \hat{\delta}_{10}, \hat{\delta}_{11}\right)
$$

The error estimation is derived every two steps. The composition method with the embedded form can be expressed in Butcher tableau

$$
\begin{array}{c|cc}
c & A & \\
1+\bar{c} & B_{1} & \bar{A} \\
\hline 0 & & \delta^{T}
\end{array},
$$

where $B_{1}=(\underbrace{b, \ldots, b}_{5})^{T}$. 
The order conditions for the one-step zero approximation up to order four and some trees of order five are given as follows:

$$
\begin{array}{llll}
d_{1}=\hat{\delta}^{T} e, & d_{2}=\hat{\delta}^{T} \hat{c}, & d_{3}=\hat{\delta}^{T} \hat{c}^{2}, & d_{4}=\hat{\delta}^{T} \hat{c}^{3}, \\
d_{5}=\hat{\delta}^{T} \hat{c}^{4}, & d_{6}=\hat{\delta}^{T} \hat{A} \hat{c}^{2}, & d_{7}=\hat{\delta}^{T} \widehat{A}^{2} \hat{c}, & d_{8}=\hat{\delta}^{T} \hat{c} \widehat{A} \hat{c}^{2},
\end{array}
$$

Solving $d_{1}=d_{2}=\cdots=d_{8}=0$, we obtain $\hat{\delta}_{1}, \hat{\delta}_{3}, \hat{\delta}_{4}, \hat{\delta}_{5}, \hat{\delta}_{6}, \hat{\delta}_{8}, \hat{\delta}_{9}, \hat{\delta}_{10}$. We choose the free parameter $\hat{\delta}_{11}=1$. The error estimation is derived (see Appendix).

The error estimation of the composition method can be regarded as linear combination of the stage values and output values over two steps.

\section{Numerical experiments}

In this section, numerical experiments were carried out on the well-known set of test problems, DETEST on interval $[0,20]$. The numerical results of the Kepler orbital problems D1 and D3 with eccentricity $=0.1$ and 0.5 respectively are shown in these numerical experiments. It is well-known that the Dormand and Prince method (DOPRI $(5,4)$ ) has outstanding performance in solving the orbit problems. Our aim is to compare the numerical behavior of the new pair composition method (ECRK5) with the famous Runge-Kutta method RK54 and the Dormand and Prince method DOPRI $(5,4)$. Firstly, experiments are set to verify that our new pair satisfying order five. The numerical results are shown in Table 2. The composition method has attained order five.

Secondly, experiments were carried out using a changing stepsize pattern in which a predetermined sequence of stepsizes was imposed [13]. For each sequence of five steps, stepsizes in the ratios 1:r:r $r^{2}: r: 1$ were used, where $r$ is a parameter. Fixed and variable stepsizes comparisons on the DETEST problems $D 1$ and $D 3$ are presented in Figure 1 which $r=1$ (fixed stepsize), $r=1.5$ and $r=2$ are given.

Table 2. Comparison of error behaviors for testing D1 and D3 (ECRK5)

\begin{tabular}{|c|c|c|c|c|c|}
\hline$h$ & D1:Truncation Error & Ratio & $h$ & D3:Truncation Error & Ratio \\
\hline 0.04 & $1.134 \times 10^{-7}$ & 33.6 & $\frac{20}{750}$ & $2.373 \times 10^{-5}$ & 29.4 \\
\hline 0.02 & $3.909 \times 10^{-9}$ & 31.93 & $\frac{20}{1500}$ & $8.071 \times 10^{-7}$ & 31.31 \\
\hline 0.01 & $1.263 \times 10^{-11}$ & & $\frac{20}{3000}$ & $2.557 \times 10^{-8}$ & \\
\hline
\end{tabular}



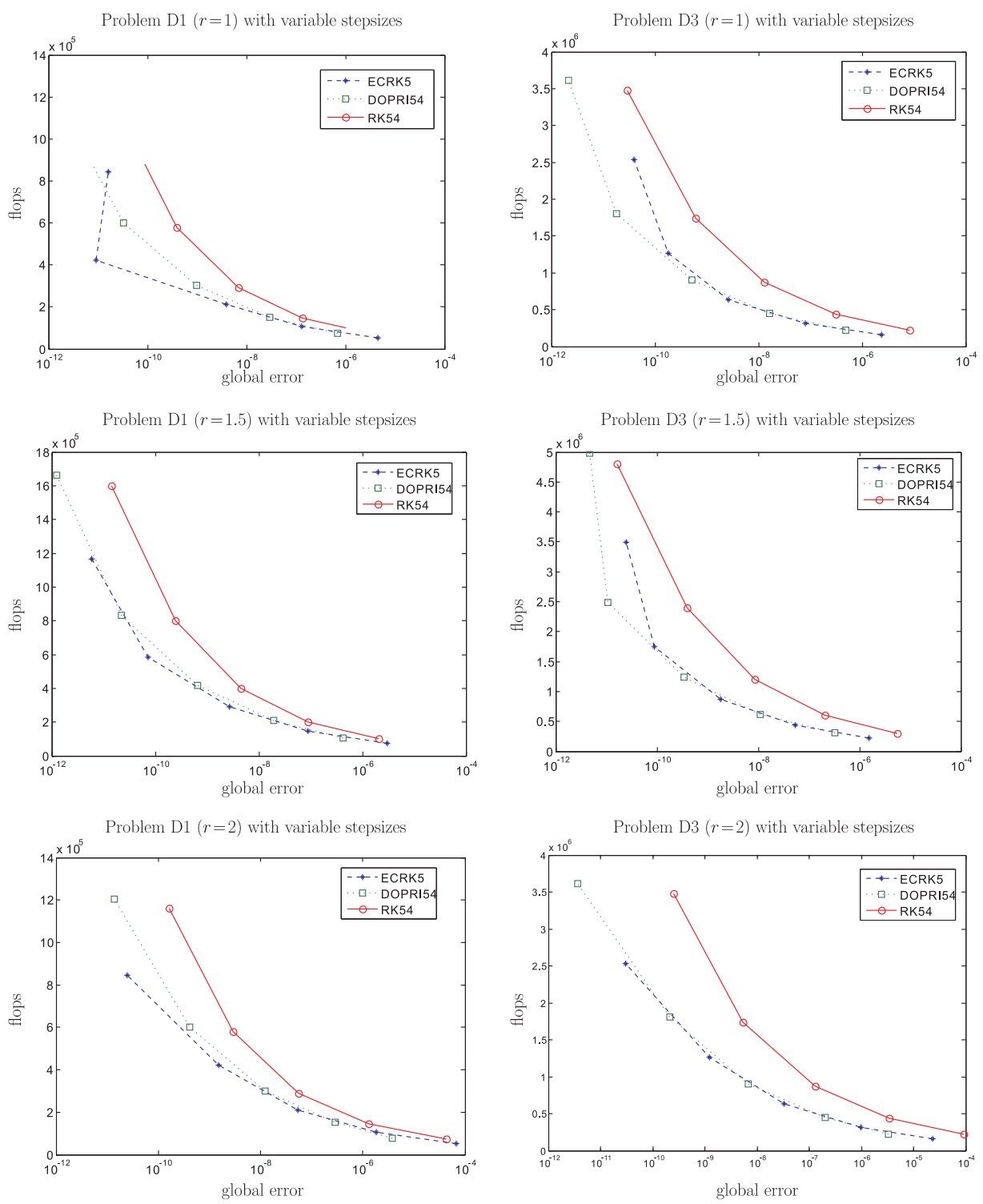

Figure 1. Fixed and variable stepsizes comparisons on the DETEST D1 and D3. 
Finally, the experiments are carried out using a changing stepsize scheme. The tolerances are chosen from $10^{-4}$ to $10^{-10}$. Numerical results are shown in Figure 2.
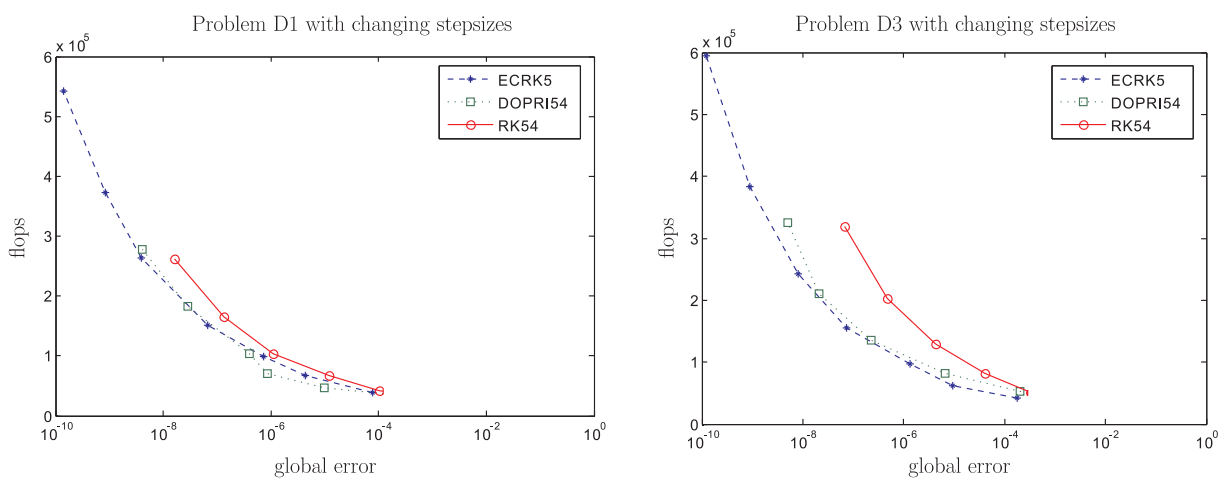

Figure 2. The numerical results for DETEST D1 and D3.

It is noted that all methods tested have order five. The ECRK5 performs more efficient than RK54, and is competitive with DOPRI $(5,4)$. ECRK5 has better error estimation may explain the numerical results.

\section{Conclusion}

In this paper, a fifth order explicit Runge-Kutta composition method with five stages is derived. A reliable error estimator is also derived by using linear combination of stage values and output values over two steps. Numerical results obtained by testing the new pair over a standard set of test problems show a significant improvement. Moreover, the composition pair also presents attainable order 5. Our future work is to apply this idea to triple composition method, diagonal implicit Runge-Kutta method and higher derivatives methods.

\section{Appendix}

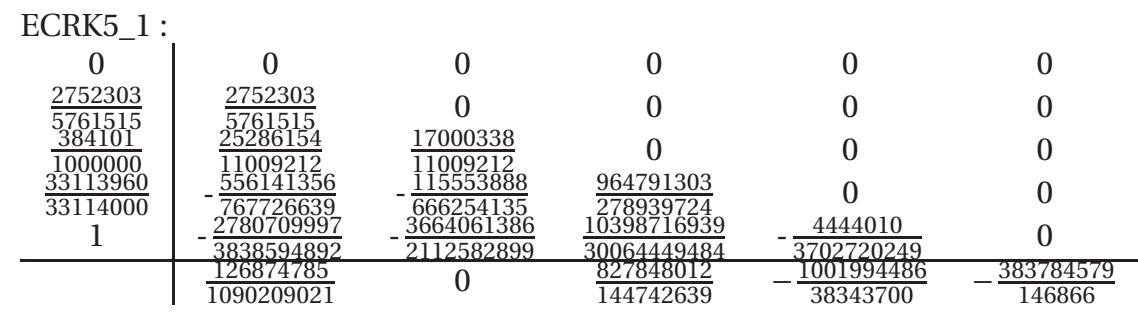




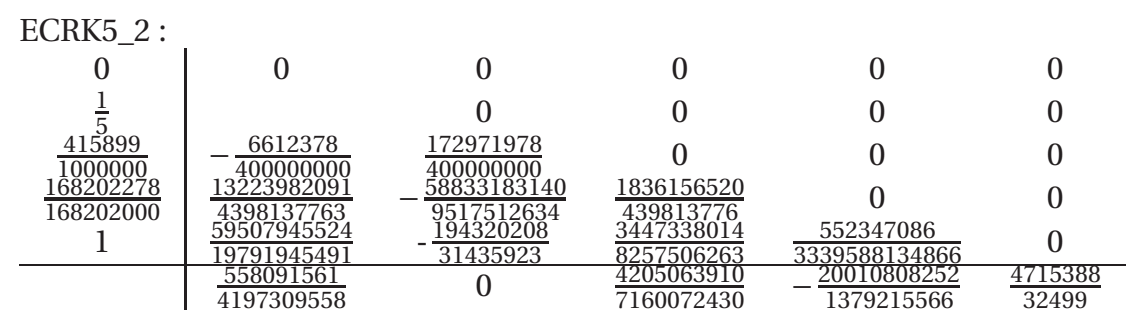

Error estimation :

$\hat{\delta}_{1}=\frac{944328183026764768228322793636375561453801003824979250183767179}{14957162936792244628752151721894194895687259064219989027007365642}$,

$\hat{\delta}_{2}=0$,

$\hat{\delta}_{3}=-\frac{8814666734001641467027839398827273093980601326261583382273500000000000}{19858019914258203296704742004601252979601984577626993781625694374802447}$

$\hat{\delta}_{4}=\frac{3993984711932193046634586456860660859758455434522338043206491571293500000000000}{5260578}$,

$\hat{\delta}_{4}=\frac{52605781927012633487676951472913330063325469100136762288196200043011}{\hat{\delta}_{5}}=-\frac{152978133143822941647581035256522250876698636937077458140184639241}{2014934111884061240552456398651833893442460778626769559494}$,

$\hat{\delta}_{5}=-\frac{1501493411188406124065524606398651833893442460778626769559494}{50}$,

$\hat{\delta}_{6}=-\frac{5590354464895655537437898942009357871060935186343610298157755708855}{575851435175285477831915148945441563586210082420744510820434516458}$,

$\hat{\delta}_{7}=0$,

$\hat{\delta}_{8}=\frac{311471582159985795458727144856463443218576652557223506938414500000000000}{98232878182364177385359281350115552001623341080668590103749995538051387}$,

$\hat{\delta}_{9}=\frac{1996795850307859889094117535056663128880641855947300041788772737207177500000000000}{1892219891055729932242033593723225669632173322568690846988894179787226985213}$,

$\hat{\delta}_{10}=-\frac{1892219891055729932242033593723225669632173322568690846988899941797872}{445878042114001612986100489882492932446691986555586074494514}$,

$\hat{\delta}_{11}=1$.

\section{References}

[1] P. Albrecht, A new theoretical approach to Runge-Kutta methods, SIAM J. Numerical Anal. 24(1987), 391-406.

[2] F. Bornemann, Runge-Kutta methods, Trees, and Mathematica, Selcuk J. Appl. Math. 2(2001), 1-13.

[3] J. C. Butcher, On the attainable order of Runge-Kutta methods, Math. Comp. 19(1965), 408-417.

[4] J. C. Butcher, The effective order of Runge-Kutta methods, Conference on the Numerical Solution of Differential Equations, Lecture Notes in Math., 109(1969), 133-139.

[5] J. C. Butcher, An Algebraic theory of integration methods, Math. Comp. 26(1972), 79-106.

[6] J. C. Butcher, An introduction to "Almost Runge-Kutta" methods, Appl. Numerical Math. 24(1997), 331-342.

[7] J. C. Butcher, Numerical Methods for Ordinary Differential Equations. John Wiley \& Sons, 2002.

[8] J. C. Butcher and T. M. H. Chan, Multi-step zero approximations for stepsize control, Applied Numerical Mathematics 34(2000), 167-177.

[9] J. C. Butcher and T. M. H. Chan, A new approach to the algebraic structures for integration methods, BIT, 42(2002), 477-489.

[10] J. C. Butcher and P. Chartier, The effective order of singly-implicit Runge-Kutta methods, Numer. Algorithms 20(1999), 269-284.

[11] J. C. Butcher and D. J. L. Chen, ESIRK methods and variable stepsize, Appl. Numerical Math. 28(1998), 193-207.

[12] J. C. Butcher and M. T. Diamantakis, Diagonally extended simply implicit Runge-Kutta effective order methods, Numer. Algorithms 17, 121-145.

[13] J. C. Butcher and N. Moir, Experiments with a new fifth order method, Numerical Algorithms 33(2003), 137-151. 
[14] A. R. Gifkins, An algebraic approach to Runge-Kutta methods, Thesis submitted for the Degree of Doctor of Philosophy at the University of Auckland, Department of Mathematics, 1972.

[15] N. Moir, ARK methods: some recent developments, Journal of Computational and Applied Mathematics 175(2005), 101-111.

[16] E. Hairer and S. P. N $\phi$ rsett and G. Wanner, Solving Ordinary Differential Equations I: Nonstiff Problems, Springer-Verlag, Berlin, 1993.

Department of Business Administration, Ling Tung University, Taichung, Taiwan.

E-mail: dchen@mail.ltu.edu.tw

Department of Information Technology, Ling Tung University, Taichung, Taiwan.

Department of Information Management, National Yunlin University of Science and Technology, Yunlin, Taiwan.

E-mail: changjc@mail.ltu.edu.tw

Department of Information Management, National Yunlin University of Science and Technology, Yunlin, Taiwan.

E-mail: chcheng@mis4k.mis.yuntech.edu.tw 